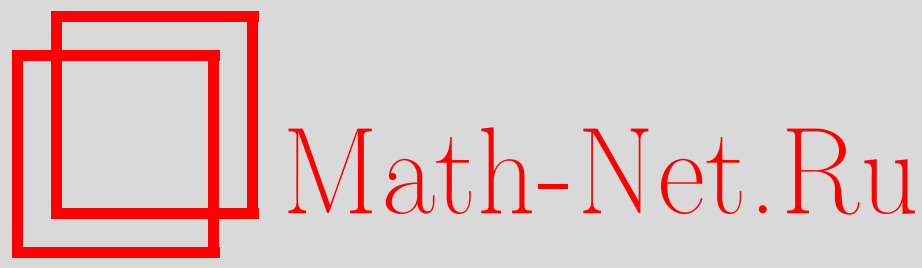

K. С. Арамян, Интегрируемая модель двумерного сингулярного сферического осциллятора в постоянном магнитном поле, ТМФ, 2008, том 156, номер 1, 131-137

DOI: https://doi.org/10.4213/tmf6234

Использование Общероссийского математического портала Math-Net.Ru подразумевает, что вы прочитали и согласны с пользовательским соглашением http://www.mathnet.ru/rus/agreement

Параметры загрузки:

IP: 18.234.197.8

26 апреля 2023 г., 03:48:17

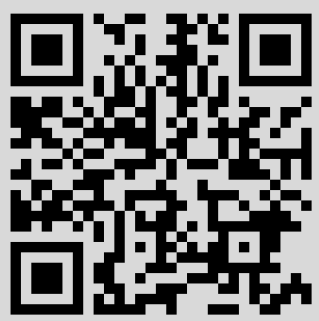




\title{
ИНТЕГРИРУЕМАЯ МОДЕЛЬ ДВУМЕРНОГО СИНГУЛЯРНОГО СФЕРИЧЕСКОГО ОСЦИЛЛЯТОРА В ПОСТОЯННОМ МАГНИТНОМ ПОЛЕ
}

\begin{abstract}
Предложен аналог двумерного сингулярного осциллятора (осциллятора Смородинского-Винтерница) на сфере, который точно решается в классическом смысле как в отсутствие, так и в присутствии постоянного магнитного поля. Найдены его явные классические решения.
\end{abstract}

Ключевые слова: сингулярный осциллятор, магнитное поле, интегрируемая модель.

\section{1. ВВЕДЕНИЕ}

Осциллятор является системой, примечательной во многих отношениях, прежде всего наличием широкой алгебры симметрий $s u(d)$, где $d$ - размерность пространства [1]. Кроме того, наряду с задачей Кулона, он отличается тем, что все его траектории замкнуты [2]. Наличие широкой алгебры симметрий позволяет во многих случаях вводить взаимодействие осциллятора с внешними полями, оставляя систему интегрируемой. Простейшим и важнейшим примером такого рода является двумерный осциллятор в постоянном магнитном поле. Наконец, имеются обобщения осциллятора на искривленные пространства. Наиболее известное обобщение такого рода было предложено Хиггсом [3] для осциллятора на $d$-мерной сфере радиуса $r_{0}$. Оно задается потенциалом

$$
V_{\mathrm{osc}}=\frac{\alpha^{2} r_{0}^{2} \operatorname{tg}^{2} \theta}{2}
$$

где $\operatorname{tg} \theta=x_{d+1} / r_{0}$, а $\mathbf{x}, x_{d+1}$ суть евклидовы координаты объемлющего пространства $\mathbb{R}^{d+1}: \mathbf{x}^{2}+x_{d+1}^{2}=r_{0}^{2}$. Наряду с обобщением задачи Кулона на сфере [4] такой осциллятор выделен тем, что все его траектории замкнуты. Отметим, что обе эти системы обладают нелинейными алгебрами скрытых симметрий, и в основном наследуют характерные свойства стандартных задач осциллятора и Кулона.

Однако недостаток осциллятора Хиггса состоит в том, что в присутствии постоянного магнитного поля он перестает быть точно решаемой системой, а это сильно уменьшает его практическую ценность. Потому важно найти другие обобщения осциллятора на сфере, остающиеся интегрируемыми в присутствии магнитного поля.

*Арцахский государственный университет, Степанакерт. E-mail: k_aramyan@rambler.ru 
В работе [5] было предложено обобщение осциллятора на комплексных проективных пространствах $\mathbb{C} P^{N}$, точно решаемое как в отсутствие, так и в присутствии постоянного магнитного поля. $\mathrm{C}$ другой стороны, $\mathbb{C} P^{1}$ (комплексная проективная плоскость) эквивалентна стереографической проекции двумерной сферы. Его квантово-механическое решение было дано в [6], [7], а классические решения построены в [8] (см. также [9]). Аналоги этой системы на четырехмерных сфере и гиперболоиде были построены, соответственно, в работах [10], [11]: они допускают включение поля инстантона Белавина-Полякова-Шварца-Тюпкина. Затем эти модели были распространены на сферы произвольной размерности [12]. Потенциал указанных моделей выглядит следующим образом:

$$
V_{\mathrm{BN}}=2 \alpha^{2} r_{0}^{2} \operatorname{tg}^{2} \frac{\theta}{2}
$$

Системы на сфере имеют не только теоретическое, но и прикладное значение. Во-первых, модель частицы на двумерной (четырехмерной) сфере, движущейся в постоянном магнитном (инстантонном) поле, является основой, на которой строится теория двумерного (четырехмерного) эффекта Холла (см., соответственно, [13], [14]). Во-вторых, системы на сферах могут интерпретироваться как системы с зависящей от положения эффективной массой частицы. Наконец, они могут аппроксимировать движение реальных частиц в тонких сферических пленках и имеют отношение к физике наноструктур. С прикладной точки зрения именно вторая модель, допускающая включение постоянного магнитного поля, особо интересна. Заметим, что под постоянным магнитным полем мы здесь имеем в виду магнитное поле, постоянное на поверхности двумерной сферы, но не в трехмерном евклидовом пространстве. Его можно интерпретировать как магнитное поле монополя Дирака, помещенного в центр сферы. Поэтому с точки зрения описания движения частиц на поверхности сферы это поле нефизично. Но если рассматривать модель как систему с зависящей от положения эффективной массой частицы, то такое поле приобретает смысл постоянного магнитного поля в общепринятом смысле.

Целью настоящей работы является исследование обобщений осциллятора на двумерной сфере в присутствии постоянного магнитного поля. А именно, мы построим сферический аналог сингулярного двумерного осциллятора (известного также как осциллятор Смородинского-Винтерница), задаваемого потенциалом [15]

$$
V_{\mathrm{SW}}=\frac{\alpha^{2} r^{2}}{2}+\frac{\beta^{2}}{2 r^{2}}, \quad r=\sqrt{x_{1}^{2}+x_{2}^{2}} .
$$

За основу сферического обобщения потенциала Смородинского-Винтерница возьмем осцилляторный потенциал на сфере, неявно содержащийся в работе [5]. Покажем, что он, естественно, расширяется до сферического аналога потенциала двумерного сингулярного осциллятора, который имеет вид

$$
V=2 \alpha^{2} r_{0}^{2} \operatorname{tg}^{2} \frac{\theta}{2}+\frac{\beta^{2}}{8 r_{0}^{2}} \operatorname{ctg}^{2} \frac{\theta}{2}
$$


Как и осциллятор Смородинского-Винтерница, построенная система остается точно решаемой в присутствии постоянного магнитного поля. Отметим, что при $\alpha^{2}=\beta^{2}$ эта система инвариантна относительно отражений.

Работа построена следующим образом. В разделе 2 найдены решения классических уравнений движения сингулярного кругового осциллятора в присутствии постоянного магнитного поля. В разделе 3 построен сферический аналог сингулярного кругового осциллятора и найдены решения его классических уравнений движения в присутствии постоянного магнитного поля. В заключении обсуждаются задачи, которые мы предполагаем решить впоследствии.

\section{2. СИНГУЛЯРНЫЙ КРУГОВОЙ ОСЦИЛЛЯТОР В ПОСТОЯННОМ МАГНИТНОМ ПОЛЕ}

Пусть мы имеем аксиально-симметричную систему на плоскости, движущуюся во внешнем постоянном магнитном поле, направленном перпендикулярно плоскости: $B_{1}=B_{2}=0, B_{3}=B$.

Если внешнее магнитное поле имеет напряженность $\mathbf{B}$, то на частицу действует сила Лоренца ${ }^{1)} \mathbf{F}_{\mathrm{L}}=\dot{\mathbf{r}} \times \mathbf{B}$. Ясно, что наличие постоянного магнитного поля не нарушает вращательной симметрии системы, а также не меняет ее энергии. Поэтому в присутствии постоянного магнитного поля двумерный осциллятор остается системой, интегрируемой по Лиувиллю. Он имеет два интеграла движения: энергию и вращательный момент. С их помощью можно проинтегрировать уравнения движения аналогично тому, как это сделано в книге Ландау и Лифшица для случая движения частицы в потенциальном центрально-симметричном поле [16].

Однако при сохранении функционального вида энергии постоянное магнитное поле меняет вид вращательного момента. Если перейти к полярным координатам, то энергия и вращательный момент сингулярного кругового осциллятора задаются выражениями

$$
E=\frac{\dot{r}^{2}+r^{2} \dot{\varphi}}{2}+\frac{\alpha^{2} r^{2}}{2}+\frac{\beta^{2}}{2 r^{2}}, \quad \mathcal{J}=r^{2} \dot{\varphi}+\frac{B r^{2}}{2} .
$$

Отсюда находим

$$
2 E=\dot{r}^{2}+\frac{\left(\mathcal{J}-B r^{2} / 2\right)^{2}}{r^{2}}+\alpha^{2} r^{2}+\frac{\beta^{2}}{r^{2}}
$$

Эквивалентно,

$$
2\left(\frac{d x}{d t}\right)^{2}=-\left(\alpha^{2}+B^{2} / 4\right) x^{2}+2(E+\mathcal{J} B / 2) x-\left(\mathcal{J}^{2}+\beta^{2}\right), \quad x \equiv r^{2} .
$$

Поэтому допустимые значения интегралов движения удовлетворяют условию

$$
(E+\mathcal{J} B / 2)^{2}>\left(\alpha^{2}+B^{2} / 4\right)\left(\mathcal{J}^{2}+\beta^{2}\right) .
$$

Из (4) находим

$$
t=\frac{1}{2} \int \frac{d x}{\sqrt{-\left(\alpha^{2}+B^{2} / 4\right) x^{2}+2(E+\mathcal{J} B / 2) x-\left(\mathcal{J}^{2}+\beta^{2}\right)}} .
$$

\footnotetext{
1) Мы пользуемся системой единиц, в которой $\mu=c=e=1$.
} 
Этот интеграл вычисляется в явном виде, в результате чего получаем [17]

$$
t=-\frac{1}{2 \sqrt{\alpha^{2}+B^{2} / 4}} \arcsin \frac{(E+\mathcal{J} B / 2)-\left(\alpha^{2}+B^{2} / 4\right) x}{\sqrt{(E+\mathcal{J} B / 2)^{2}-\left(\alpha^{2}+B^{2} / 4\right)\left(\mathcal{J}^{2}+\beta^{2}\right)}}
$$

или, эквивалентно,

$$
r^{2}=\frac{E+\mathcal{J} B / 2}{\alpha^{2}+B^{2} / 4}\left(1+\sqrt{1-\frac{\left(\alpha^{2}+B^{2} / 4\right)\left(\mathcal{J}^{2}+\beta^{2}\right)}{(E+\mathcal{J} B / 2)^{2}}} \sin 2 \sqrt{\alpha^{2}+B^{2} / 4} t\right) .
$$

Как видим, наличие центробежного потенциала не влияет на частоту радиальных колебаний, а влияет только на их амплитуду.

Принимая во внимание, что вращательный момент имеет вид $\mathcal{J}=x \dot{\varphi}+B x / 2$, мы можем найти также зависимость $\varphi=\varphi(t)$ :

$$
\varphi=\int d t \frac{\mathcal{J}-B x / 2}{x}=-\frac{B t}{2}+\mathcal{J} \int \frac{d t}{r^{2}(t)} .
$$

Этот интеграл тоже вычисляется [17], в результате окончательно получаем

$$
\begin{aligned}
& \frac{\sqrt{\left(\alpha^{2}+B^{2} / 4\right)\left(\mathcal{J}^{2}+\beta^{2}\right)}}{E+\mathcal{J} B / 2} \operatorname{tg}\left(\mathcal{J}+\beta^{2} / \mathcal{J}\right)(\varphi+B t / 2)= \\
& =\sqrt{1-\frac{\left(\alpha^{2}+B^{2} / 4\right)\left(\mathcal{J}^{2}+\beta^{2}\right)}{(E+\mathcal{J} B / 2)^{2}}}+\operatorname{tg} \sqrt{\alpha^{2}+B^{2} / 4} t .
\end{aligned}
$$

Итак, мы показали, что двумерный сингулярный осциллятор в постоянном магнитном поле является классически точно решаемой системой. Мы нашли его явные решения и убедились, что наличие центробежного члена качественно не меняет свойства системы, хотя и влияет на вид решений уравнений движения. Поэтому можно ожидать, что и в случае сферического сингулярного осциллятора будет иметь место аналогичное явление.

\section{3. СФЕРИЧЕСКИЙ СИНГУЛЯРНЫЙ ОСЦИЛЛЯТОР}

Как построить сферический аналог осциллятора Смородинского-Винтерница? Прежде чем приступить к обсуждению этого вопроса, напомним основные элементы сферической геометрии. Метрика на двумерной сфере задается выражением

$$
d s^{2}=r_{0}^{2}\left(d \theta^{2}+\sin ^{2} \theta d \varphi^{2}\right),
$$

где $\theta$ и $\varphi$ связаны с евклидовыми координатами сферы следующим образом:

$$
x_{1}=r_{0} \sin \theta \sin \varphi, \quad x_{2}=r_{0} \sin \theta \cos \varphi, \quad x_{3}=r_{0} \cos \theta .
$$

В пределе $r_{0} \rightarrow \infty$ метрика сферы переходит в метрику плоскости. Для аналогии с рассмотренным ранее осциллятором на плоскости удобно воспользоваться стереографической проекцией сферы на плоскость, касательную южному полюсу сферы. Тогда каждой точке сферы $(\theta, \varphi)$ соответствует точка $u=y_{1}+i y_{2}$ плоскости

$$
y_{1}=2 r_{0} \operatorname{ctg} \frac{\theta}{2} \sin \varphi, \quad y_{2}=2 r_{0} \operatorname{ctg} \frac{\theta}{2} \cos \varphi .
$$


Соответственно

$$
r=\sqrt{y_{1}^{2}+y_{2}^{2}}=2 r_{0} \operatorname{ctg} \frac{\theta}{2} .
$$

Рассмотрим пространственное отражение

$$
\mathbf{r} \rightarrow-\mathbf{r} \Longleftrightarrow \theta \rightarrow \pi-\theta, \varphi \rightarrow-\varphi
$$

Ясно, что при этом преобразовании метрика сферы (и кинетическая энергия частицы на сфере) не меняется. Потенциал осциллятора Хиггса (1) также инвариантен относительно указанного преобразования, а потенциал (2) неинвариантен относительно этого преобразования. Он преобразуется следующим образом:

$$
2 \alpha^{2} r_{0}^{2} \operatorname{ctg}^{2} \frac{\theta}{2} \rightarrow 2 \alpha^{2} r_{0}^{2} \operatorname{tg}^{2} \frac{\theta}{2}
$$

Эквивалентно,

$$
\alpha^{2} r^{2} \rightarrow \frac{\alpha^{2}}{r^{2}}
$$

Поэтому, естественно ввести следующий сферический аналог потенциала осциллятора Смородинского-Винтерница:

$$
V_{\mathrm{BN}}=2 \alpha^{2} r_{0}^{2} \operatorname{tg}^{2} \frac{\theta}{2}+\frac{\beta^{2}}{8 r_{0}^{2}} \operatorname{ctg}^{2} \frac{\theta}{2}
$$

Коэффициенты мы подобрали так, чтобы в плоском пределе получался потенциал Смородинского-Винтерница (3). Соответственно энергия предложенной нами модели сингулярного осциллятора задается выражением

$$
E=\frac{r_{0}^{2} \dot{\theta}^{2}}{2}+\frac{r_{0}^{2} \dot{\varphi}^{2} \sin ^{2} \theta}{2}+2 \alpha^{2} r_{0}^{2} \operatorname{tg}^{2} \frac{\theta}{2}+\frac{\beta^{2}}{8 r_{0}^{2}} \operatorname{ctg}^{2} \frac{\theta}{2}
$$

При свободном движении на сфере вращательный момент задается выражением $J=r_{0}^{2} \dot{\varphi} \sin ^{2} \theta$.

При наличии вращательно-инвариантного потенциала, в том числе потенциала, соответствующего уравнению (4), эта величина опять сохраняется. Воспользовавшись приведенными выражениями энергии и вращательного момента, мы можем проинтегрировать уравнения движения. Однако мы рассмотрим более общую задачу, когда система помещена в постоянное магнитное поле.

В присутствии постоянного магнитного поля вращательный момент частицы на сфере задается выражением (см., например, [8])

$$
\mathcal{J}=r_{0}^{2} \dot{\varphi} \sin ^{2} \theta-B r_{0}^{2}(1-\cos \theta)
$$

Теперь мы готовы к интегрированию уравнений движения. Как и в предыдущем разделе, воспользуемся для этого законами сохранения. А именно, мы исключим из выражения для энергии (6) угловую скорость $\dot{\varphi}$, воспользовавшись выражением для углового момента (7). В результате получаем

$$
\frac{2 E}{r_{0}^{2}}=\left(\frac{d \theta}{d t}\right)^{2}+\frac{\left(\mathcal{J} / r_{0}^{2}+B(1-\cos \theta)\right)^{2}}{1-\cos ^{2} \theta}+4 \alpha^{2} \frac{1-\cos \theta}{1+\cos \theta}+\frac{\beta^{2}}{4 r_{0}^{4}} \frac{1+\cos \theta}{1-\cos \theta}
$$


Это выражение можно представить в следующем виде:

$$
\left(\frac{d x}{d t}\right)^{2}=M+2 N x+K x^{2}
$$

где

$$
\begin{gathered}
x=1-\cos \theta, \\
M=\frac{\mathcal{J}^{2}+\beta^{2}}{r_{0}^{4}}, \quad N=\frac{2 E+\mathcal{J} B}{r_{0}^{2}}-\frac{\beta^{2}}{4 r_{0}^{4}}, \quad K=4 \alpha^{2}+B^{2}+\frac{\beta^{2}}{4 r_{0}^{4}}-\frac{2 E}{r_{0}^{2}} .
\end{gathered}
$$

Отсюда получаем табличный интеграл [17]

$$
t=\int \frac{d x}{\sqrt{M+2 N x+K x^{2}}}=-\frac{1}{\sqrt{-K}} \arcsin \frac{K x+N}{\sqrt{N^{2}-K M}} .
$$

Окончательно, зависимость угла $\theta$ ("радиуса") от времени определяется выражением

$$
1-\cos \theta=-\Lambda \sin \sqrt{K} t, \quad \Lambda \equiv \frac{N}{K}\left(1+\sqrt{1-K M / N^{2}}\right) .
$$

Таким образом, в отличие от плоского сингулярного осциллятора, эффективная частота сферического сингулярного осциллятора $\sqrt{K} / 2$ зависит от параметра сингулярности $\beta$. Как и следовало ожидать, частота колебаний сферического сингулярного осциллятора зависит симметричным образом от параметров $2 \alpha r_{0}$ (исходной частоты) и $\beta / 2 r_{0}$. Как и в случае несингулярного сферического осциллятора, частота радиальных колебаний зависит от энергии системы. Иными словами, в отличие от плоской системы, колебания сферической системы негармоничны.

Теперь найдем зависимость от времени угловой переменной $\varphi$. Из (7) получаем

$$
\varphi=\int d t \frac{\mathcal{J} / r_{0}^{2}+B(1-\cos \theta)}{1-\cos ^{2} \theta}=\left(\frac{\mathcal{J}}{2 r_{0}^{2}}+B\right) \int \frac{d t}{1+\cos \theta}+\frac{\mathcal{J}}{2 r_{0}^{2}} \int \frac{d t}{1-\cos \theta} .
$$

Подставив сюда выражение (8), вновь получим табличный интеграл [17], который даст нам явную зависимость угловой переменной от времени:

$$
\varphi=\frac{\mathcal{J}}{r_{0}^{2} \sqrt{K} \sqrt{1-\Lambda^{2}}} \operatorname{arctg} \frac{\operatorname{tg} \sqrt{K} t / 2-\Lambda}{\sqrt{1-\Lambda^{2}}}+\frac{\mathcal{J} / r_{0}^{2}+2 B}{\sqrt{K} \sqrt{1-\Lambda^{2} / 4}} \operatorname{arctg} \frac{\operatorname{tg} \sqrt{K} t / 2+\Lambda / 2}{\sqrt{1-\Lambda^{2} / 4}} .
$$

Как видим, уравнения движения угловой переменной также явно интегрируются. Заметим, что в отличие от плоского сингулярного случая, зависимость эволюции угла от магнитного поля гораздо более сложная.

\section{4. ЗАКЛЮЧЕНИЕ}

В настоящей работе мы предложили сферическое обобщение двумерного сингулярного осциллятора (осциллятора Смородинского-Винтерница) и рассмотрели его поведение в постоянном магнитном поле. Мы показали, что эта система точно решаема на классическом уровне, предъявив ее явные классические решения. 
Вне всякого сомнения, предложенная система является также точно решаемой на квантовом уровне: ее квантово-механические решения можно получить из решений сферического (несингулярного) осциллятора [6] соответствующим переопределением магнитного квантового числа $|m| \rightarrow \sqrt{m^{2}+\beta^{2}}$. Хотя способ получения квантово-механических решений очевиден, они заслуживают более детального анализа. Так, интересно посмотреть свойства спектра системы при $2 \alpha r_{0}=\beta / 2 r_{0}$, т.е. в случае, когда система обладает симметрией относительно пространственных отражений. Наконец, интересно проанализировать оптические свойства предложенной модели с целью выяснения возможности ее применения в описании кольцеобразных квантовых точек.

Благодарности. Автор благодарен А. Нерсесяну за предложенную задачу и помощь в оформлении рукописи.

\section{Список литературы}

[1] А. М. Переломов, Интегрируемые системы классической механики и алгебры Ли, Наука, М., 1990.

[2] В. И. Арнольд, Математические методъ классической механики, Наука, М., 1989.

[3] P. W. Higgs, J. Phys. A, 12:3 (1979), 309-323; H. I. Leemon, J. Phys. A, 12:4 (1979), 489-501.

[4] E. Schrödinger, Proc. Roy. Irish Soc., 46 (1940), 9-16; 46 (1941), 183-206; 47 (1941), $53-54$.

[5] S. Bellucci, A. Nersessian, Phys. Rev. D, 67:6 (2003), 5013.

[6] S. Bellucci, A. Nersessian, A. Yeranyan, Phys. Rev. D, 70:8 (2004), 5013.

[7] S. Bellucci, A. Nersessian, A. Yeranyan, Phys. Rev. D, 70:4 (2004), 5006.

[8] M. A. Alexanyan, K. S. Aramyan, J. Contemp. Phys., 42:2 (2007), 49-54.

[9] A. Nersessian, A. Yeranyan, J. Phys. A, 37:7 (2004), 2791-2801.

[10] L. Mardoyan, A. Nersessian, Phys. Rev. B, 72:23 (2005), 3303.

[11] S. Bellucci, L. Mardoyan, A. Nersessian, Phys. Lett. B, 636:2 (2006), 137-141.

[12] L. Mardoyan, A. Nersessian, A. Yeranyan, Phys. Lett. A, 366:1-2 (2007), 30-35.

[13] R. B. Laughlin, Phys. Rev. Lett., 50:18 (1983), 1395-1398.

[14] S. C. Zhang, J. P. Hu, Science, 294:5543 (2001), 823-828.

[15] П. Винтернитц, Я.А. Смородинский, М. Углирж, И. Фриш, ЯФ, 4 (1967), 625-635; J. Fris, V. Mandrasov, Ya. A. Smorodinsky, M. Uhlir, P. Winternitz, Phys. Lett., 16:3 (1965), 354-356.

[16] Л. Д. Ландау, Е. М. Лифшиц, Механика, Наука, М., 1973.

[17] Г. Б. Двайт, Таблицы интегралов и другие математические формуль, Наука, М., 1983. 